\title{
Pulmonary endarterectomy: the potentially curative treatment for patients with chronic thromboembolic pulmonary hypertension
}

\author{
David Jenkins \\ Affiliation: Dept of Cardiothoracic Surgery, Papworth Hospital, Cambridge, UK. \\ Correspondence: David Jenkins, Dept of Cardiothoracic Surgery, Papworth Hospital, Cambridge, CB23 3RE, \\ UK. E-mail: david.jenkinsapapworth.nhs.uk \\ ABSTRACT Pulmonary endarterectomy (PEA) is the treatment of choice to relieve pulmonary artery \\ obstruction in patients with chronic thromboembolic pulmonary hypertension (CTEPH). It is a complex \\ surgical procedure with a simple principle: removal of obstructive thromboembolic material from the \\ pulmonary arteries in order to reduce pulmonary vascular resistance, relieve pulmonary hypertension $(\mathrm{PH})$ \\ and alleviate right ventricular dysfunction. In the majority of patients there is symptomatic and prognostic \\ benefit. However, not all patients with CTEPH are suitable for treatment with PEA. Operability assessment is \\ not always easy, being largely subjective and based on experience. It is therefore important that all patients \\ are referred to an experienced CTEPH centre for careful evaluation of suitability for surgery. The most \\ common reason for inoperability is distal vasculopathy accounting for a high proportion of the vascular \\ resistance. Surgery requires cardiopulmonary bypass and periods of deep hypothermic circulatory arrest. \\ Complications include reperfusion lung injury and persistent $\mathrm{PH}$. However, with careful patient selection, \\ surgical technique and post-operative management, PEA is a highly effective treatment with mortality rates \\ $<5 \%$ at experienced centres. Patients who are unsuitable for surgery may be candidates for medical therapy.
}

@ERSpublications

A review of pulmonary endarterectomy as a CTEPH treatment, including patient assessment, risks and outcomes http://ow.ly/L82F7

\section{Introduction}

Pulmonary endarterectomy (PEA) is the treatment of choice to relieve pulmonary artery obstruction in patients with chronic thromboembolic pulmonary hypertension (CTEPH), and offers a potentially curative treatment for some patients [1-3]. Currently, only a few surgical centres have expert experience in performing PEA with case volumes of $>1000$ operations, but experience is growing at many centres. Most surgical experience in PEA comes from the University of California, San Diego (UCSD), USA, with $>3000$ procedures performed to date [4]. The procedure itself is technically demanding, and requires specialist training and sophisticated intensive care post-operatively $[1,5]$. However, with careful patient selection, meticulous surgical technique and post-operative management, PEA is an effective and highly successful procedure with experienced high-volume centres now reporting in-hospital mortality rates of $<5 \%$ [3].

\section{Assessment for operability}

Pre-operative risk stratification is important in patients with CTEPH, but unlike cardiac surgery, for which there is the European System for Cardiac Operative Risk Evaluation (EuroSCORE), there is no comparable objective assessment tool for CTEPH. To be considered operable, a patient must have sufficient surgically

Received: Feb 062015 | Accepted after revision: March 302015

Conflict of interest: Disclosures can be found alongside the online version of this article at err.ersjournals.com

Provenance: Publication of this peer-reviewed article was sponsored by Bayer Pharma AG, Berlin, Germany (principal sponsor, European Respiratory Review issue 136).

Copyright OERS 2015. ERR articles are open access and distributed under the terms of the Creative Commons Attribution Non-Commercial Licence 4.0. 
accessible thromboembolic material, with a proportional pulmonary vascular resistance (PVR) indicating the absence of extensive distal disease [6]. Thromboembolic disease located proximally in the main, lobar or segmental arteries can be removed relatively easily by PEA, while distal disease confined to subsegmental vessels is more difficult to clear and may therefore render the patient inoperable [2]. The final decision regarding operability is based on the risk/benefit ratio for the patient and is ultimately determined by the surgeon's experience.

Pulmonary angiography is currently regarded as the gold-standard imaging technique in evaluating the location and extent of disease, and thus suitability of the patient for PEA [2], but is only useful if performed properly with selective contrast injection and viewed in at least two planes. Advances in other imaging modalities, specifically computed tomography pulmonary angiography (CTPA) [7] and magnetic resonance imaging, have led to their increased use in conjunction with pulmonary angiography in the assessment of operability at experienced centres [2].

Before deciding whether to operate, the presence and severity of any haemodynamic impairment should be evaluated [8] and correlated with the degree of thromboembolic disease seen on imaging. The extent of haemodynamic impairment is evaluated by right heart catheterisation [2]. Most patients considered for PEA have severe haemodynamic abnormalities at rest. However, those with less severe abnormalities also warrant consideration for surgery in order to improve symptoms and possibly prevent the development of progressive pulmonary hypertension $(\mathrm{PH})$ [8]. Indeed, a small series of carefully selected patients with chronic thromboembolic disease, but no $\mathrm{PH}$ at rest (as determined by right heart catheterisation), has recently been published, which demonstrated symptomatic and quality of life benefits in this patient cohort post-PEA [9]. In the absence of notable abnormalities at rest, patients whose PVR increases markedly upon exercise, or in whom significant exercise impairment is observed, should therefore be considered for PEA [8]. Additional considerations as to whether or not to operate include the impact of patient comorbidities on peri- and post-operative risk [8]. For example, surgery is contraindicated in patients with concomitant severe parenchymal disease, since this condition has the potential to worsen ventilation/perfusion mismatch and exacerbate hypoxaemia, such that improvement in symptoms is minimal after surgery even if perfusion is improved [10]. These factors considered together allow a decision on operability to be made, with patient consent being the final requirement for surgery.

Importantly, a patient should not be considered inoperable until reviewed by a specialist CTEPH team, including an experienced PEA surgeon [1,2]. A scoring system for evaluation of operative risk, morbidity and long-term outcome would be invaluable; however, attempts to develop such a score have demonstrated that the most influential factor is the experience of the surgeon, as well as interpretation of available imaging, and that the assessment of operability is therefore somewhat subjective. The treatment decision for patients with CTEPH should be made at an experienced centre based upon interdisciplinary discussion among internists, radiologists and expert surgeons (the "CTEPH team"). Historically, some have suggested that to be considered "expert" a centre should perform at least 20 PEA operations per year with $<10 \%$ mortality rate [1].

Despite the need for careful pre-operative assessments, it is important not to delay surgery in suitable patients. Time to referral for PEA surgery may be significantly delayed for several reasons, including diagnostic difficulties, increasing use of medical therapy and centre availability. Importantly, the use of medical therapy as a bridge to surgery is unproven and should not delay surgical intervention [11].

\section{Assessing operability: lessons from the CHEST study}

The phase III, randomised, placebo-controlled Chronic Thromboembolic Pulmonary Hypertension Soluble Guanylate Cyclase-Stimulator Trial (CHEST) study of riociguat [12] used a unique methodology, in which all patients considered "inoperable" by study investigators were required to be referred for central or local adjudication by an experienced surgeon, based on recommended scanning techniques, additional haemodynamic investigations and assessment of medical history. Technical operability was assessed on the basis of surgical accessibility of organised thrombi and concordance between degree of thromboembolic disease and PVR [12]. Assessment was based on at least a pulmonary angiogram supplemented by a ventilation/perfusion scan (preferred method) or alternatively CTPA (at least 64-slice spiral computed tomography with contrast medium) with ventilation/perfusion scan (fig. 1) [12]. This ensured that patients most likely to benefit from pharmacological therapy (with distal disease) were included in the study, and those who might benefit from potentially curative PEA surgery were identified. This method may serve as a model for future CTEPH studies, as well as for the assessment of operability in clinical practice.

\section{PEA surgical procedure}

PEA surgery involves clearing all obstructive thromboembolic material from the pulmonary arteries, including the intima and superficial media (fig. 2). The aim is to reduce the PVR, to ameliorate right 

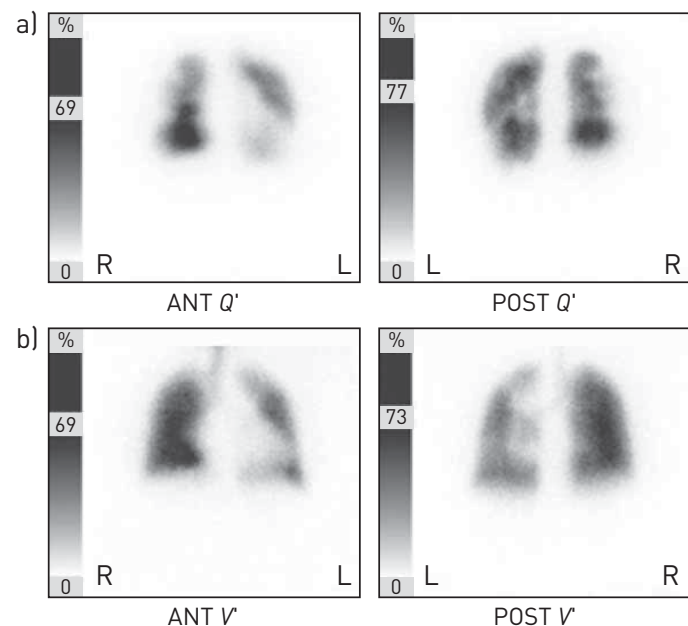

$\mathrm{R}$

POST V

c)

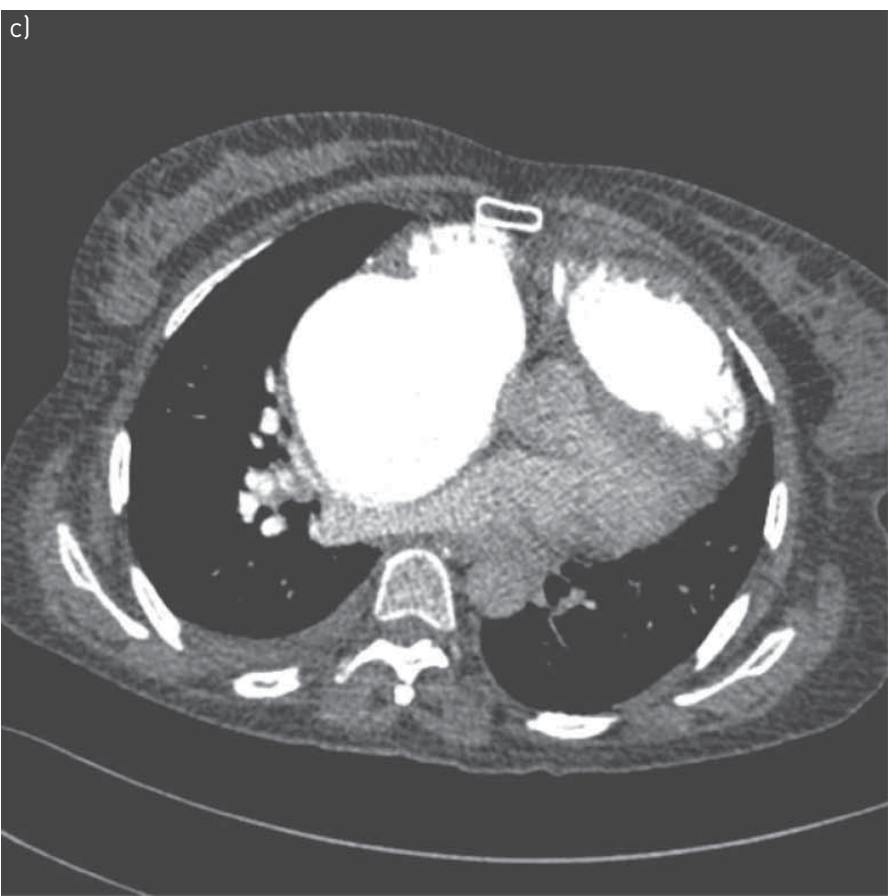

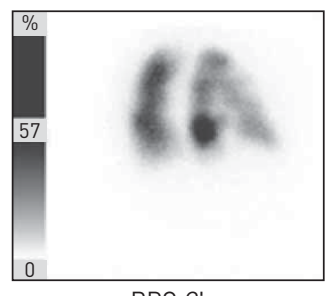

RPOQ $Q^{\prime}$

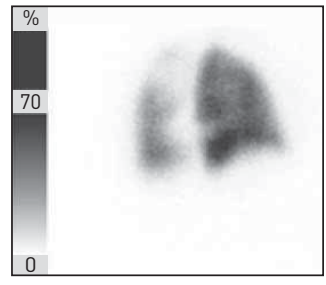

RPO $V$

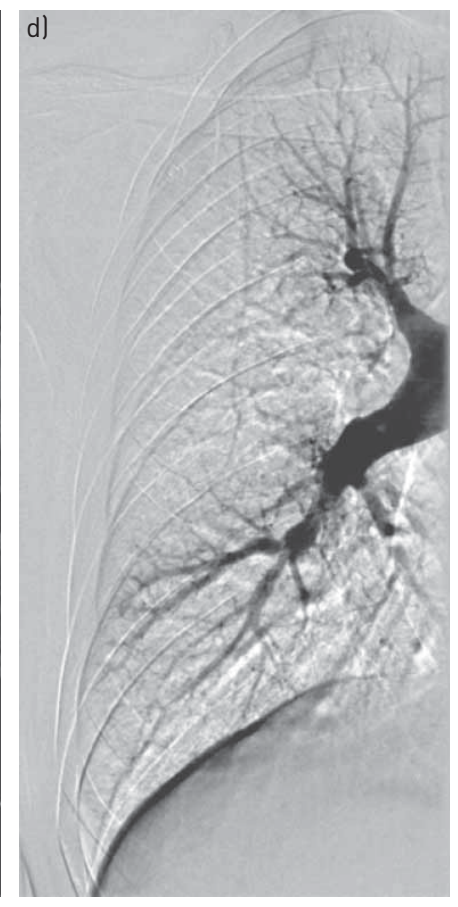

FIGURE 1 Example scans for a typical patient with operable chronic thromboembolic pulmonary hypertension. a) Perfusion $\left(Q^{\prime}\right)$ and b) ventilation $\left(V^{\prime}\right)$ lung scans. R: right; L: left; ANT: anterior; POST: posterior; LPO: left posterior oblique; RPO: right posterior oblique. c) Computed tomography pulmonary angiography scan. d) Pulmonary angiogram.

ventricular compromise and to improve ventilation/perfusion matching, although the latter may still be impaired in the early post-operative period [13].

The operation is performed through a median sternotomy incision to approach both lungs, with cardiopulmonary bypass (CPB) (established by ascending aortic and caval cannulation) enabling hypothermia to $20^{\circ} \mathrm{C}$ and safe arrest of the circulation [2]. Deep hypothermic circulatory arrest (DHCA) provides a clear operating field, such that a complete endarterectomy with dissection into subsegmental branches can be performed [2]. DHCA is limited to 20-min intervals (approximate time of unilateral PEA) and is initiated when blood obscures the surgical field. Cooling to $20^{\circ} \mathrm{C}$ must be carried out gradually over 60-90 min, to ensure uniform cooling; this can be achieved by the CPB machine, helped by use of both a cooling blanket and a head jacket [13]. Typically, right arteriotomy is performed first, and the endarterectomy plane is identified, followed by progressive dissection distally to remove the endarterectomy specimen $[14,15]$. Identification of the correct endarterectomy plane is crucial: if it is too deep, the pulmonary artery may be perforated, and if too shallow, may not permit adequate removal of thromboembolic material [13]. The ideal layer leaves a pearly white smooth residual vessel wall and the 

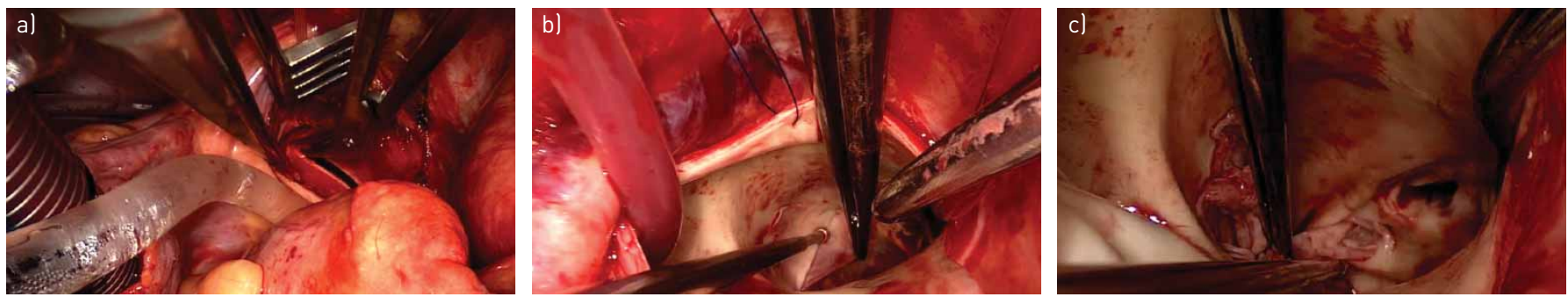

FIGURE 2 a) Longitudinal incision in the right pulmonary artery, exposed between the superior vena cava and aorta. b) Developing the endarterectomy dissection plane in the left pulmonary artery. c) Removing the occluded endarterectomy "tails" from the left upper lobe segmental vessels.

easiest dissection plane [13]. The procedure is bilateral, and on completion of the right PEA, bypass is resumed and the patient reperfused while the arteriotomy is closed so that the procedure can be repeated on the left side, with circulatory arrest being initiated as necessary [13].

\section{Potential for peri-operative complications}

The main aspects of peri-operative care and complications are similar to those of conventional heart surgery [16]. Complications more specific to PEA, occurring during or shortly after surgery, include reperfusion lung injury and neurological complications. Persistent $\mathrm{PH}$ in the immediate post-operative period also adversely influences post-operative morbidity and mortality, and will be covered in a later section of this review.

\section{Reperfusion lung injury}

The most common immediate post-operative complication that is specific to PEA is reperfusion lung injury $[16,17]$. Reperfusion lung injury typically occurs within $48 \mathrm{~h}$ of PEA surgery and is characterised by a high permeability oedema resulting in hypoxia and new radiological opacity in areas of the lungs that have been reperfused [17]. It was reported to occur in $9.6 \%$ of patients in a large European/Canadian registry [18]. Early treatment with diuresis, maintenance of haematocrit and peak end-expiratory pressure can minimise the development of oedema but, when severe, veno-venous extracorporeal membrane oxygenation (ECMO) may be required for temporary support in some of the sickest patients [16]. With ECMO, one-third to one-half of patients can be salvaged and leave hospital alive [19].

There is some preliminary evidence from a single centre that pre-operative transcatheter occlusion of the bronchopulmonary collateral artery (PTOBPCA) may reduce reperfusion pulmonary oedema after PEA in patients with CTEPH. In a recent retrospective study of 155 patients with CTEPH, a significantly lower proportion of patients in the PTOBPCA group (1.5\% (one out of 68 )) required ECMO for reperfusion pulmonary oedema after PEA than in the control group (10.3\% (nine out of 87)) (Chi-squared test 4.980; $\mathrm{p}=0.026$ ) [20]. In addition, patients who received PTOBPCA had shorter intubation and intensive care hospitalisation times and better post-PEA haemodynamics than patients in the control group.

On the basis that peri-operative corticosteroids have been shown to reduce complement activation and cytokine release in patients undergoing $\mathrm{CPB}$, the efficacy of methylprednisolone in reducing the incidence or severity of reperfusion lung injury has been assessed in a double-blind, prospective, placebo-controlled study of 98 patients with CTEPH undergoing PEA [17]. In this study, reperfusion lung injury was defined as the presence of hypoxaemia (arterial oxygen tension/inspiratory oxygen fraction $<300 \mathrm{mmHg}$ ) and new lung infiltrate in an area that had been reperfused, occurring in the absence of any other possible cause of hypoxaemia or chest radiograph abnormality on days 1-3 of the study [17]. Treatment with methylprednisolone had no significant impact on clinical outcomes and the incidence of lung injury was $41 \%$ in the corticosteroid-treated group versus $45 \%$ in the placebo group ( $\mathrm{p}=0.727$ ) [17]. Furthermore, methylprednisolone had no significant impact on the secondary end-points (number of ventilator-free, intensive care unit-free and hospital-free days), despite a reduction in pro-inflammatory cytokines [17].

\section{Neurological complications}

There is potential for neurological injury as a result of brain ischaemia during DHCA [21]. Most large contemporary series of patients undergoing PEA have reported very few clinical neurological events and the risk of stroke is low. However, because of the long period on CPB and DHCA, there is the potential for more subtle cognitive impairment and until recently the post-operative risk of cognitive decline was unquantified. In the Circulatory Arrest Versus Cerebral Perfusion During Pulmonary Endarterectomy Surgery (PEACOG) study, patients were randomised to undergo PEA with DHCA or with antegrade cerebral perfusion (ACP), where brain blood flow was maintained. The group conducting the study had previously reported the 
feasibility of using ACP to perform PEA [22]. Cognitive function was assessed before and then 3 months and 1 year after surgery [23]. This prospective controlled trial was the first to directly compare DHCA and ACP in any surgical procedure [2]. Nine patients (out of 39 randomised to undergo ACP) had to cross over to DHCA because the operating field was not clear without complete circulatory arrest [23]. Furthermore, there were no differences in cognitive function between the DHCA and ACP groups after surgery, and in fact, mean scores improved rather than declined, which could be attributed to improved cardiac output and oxygenation following PEA [23]. Thus, although some reports have suggested that PEA is possible without complete DHCA $[22,24]$, there is little evidence to support the superiority of any alternative techniques [4]. The PEACOG study confirmed that DHCA, as used for PEA surgery, is well tolerated without cognitive decline. Therefore, PEA with DHCA remains the recommended procedure [2].

\section{Inferior vena cava filters}

The rationale for inferior vena cava filters in patients undergoing PEA is uncertain and many surgical centres have now abandoned this protocol without any changes in patient outcome. Guidelines from the British Committee for Standards in Haematology recommended that the use of inferior vena cava filters is considered in patients with CTEPH undergoing PEA, based on the findings of a small case series that identified inadequate caval filtration as a common abnormality in patients requiring repeat PEA [25]. However, the committee acknowledged that the level of evidence supporting this use is low. Acute complications are rare but include filter misplacement, pneumothorax, haematoma, air embolism, carotid artery puncture and arteriovenous fistula. Potential later complications include recurrent deep vein thrombosis, inferior vena cava thrombosis or penetration, post-thrombotic syndrome and migration of the filter (which can result in entrapment of guidewires) [21].

\section{Effectiveness of PEA and survival rates}

Pulmonary occlusive disease has been classified by JAMIESON and KAPELANSKI [26] (table 1). It is important to note that this is an intra-operative classification and the distinction between more distal segmental diseases is not always clear. Surgery is generally most effective in patients with type 1 and 2 disease; however, in experienced units many patients with type 3 disease benefit from PEA, even if there is some persistent $\mathrm{PH}$ [16]. In this regard, a recent study of 331 endarterectomies that classified patients into two groups (type 1 and 2 versus type 3 disease) reported in-hospital mortality of 6.9\%, which did not differ between groups [28].

\section{Improvement in haemodynamics following PEA}

Effective PEA intervention can result in near normalisation of pulmonary haemodynamics, with significant and immediate reductions in PVR and mean pulmonary arterial pressure (mPAP), together with an increase in cardiac index (table 2). A large series at UCSD reported a post-operative reduction in PVR from $861.2 \pm 446.2$ to $294.8 \pm 204.2 \mathrm{dyn} \cdot \mathrm{s} \cdot \mathrm{cm}^{-5}$ and a reduction in mPAP from $46.1 \pm 11.4$ to $28.7 \pm 10.1 \mathrm{mmHg}$ in a cohort of 1000 patients studied [4]. In a series from the UK of 314 patients with CTEPH undergoing PEA, mPAP was reduced from $48 \pm 12 \mathrm{mmHg}$ to $26 \pm 10 \mathrm{mmHg}$ [29]. There are fewer longer-term follow-up studies, as most series report in-hospital data, but it appears that the early haemodynamic benefits remain unchanged over the medium term. For example, in a prospective evaluation of 157 patients, PVR before surgery was reported as $1140 \pm 517$, reduced to $327 \pm 238$ at 3 months and $339 \pm 251 \mathrm{dyn} \cdot \mathrm{s} \cdot \mathrm{cm}^{-5}$ at 4 years following PEA [30].

\begin{tabular}{|c|c|c|}
\hline Type & Description & Operable? \\
\hline 1 & $\begin{array}{l}\text { Major vessel clot } \\
\text { Readily visible on opening pulmonary arteries } \\
20 \% \text { of cases }\end{array}$ & Yes \\
\hline 2 & $\begin{array}{l}\text { Only thickened intima can be seen } \\
\text { Endarterectomy plane initially raised in the main, lobar or segmental vessels } \\
\sim 70 \% \text { of cases }\end{array}$ & Yes \\
\hline 3 & $\begin{array}{l}\text { Distal disease } \\
\text { Confined to segmental and subsegmental branches } \\
\sim 10 \% \text { of cases }\end{array}$ & Yes \\
\hline 4 & $\begin{array}{l}\text { Intrinsic small-vessel disease } \\
\text { Secondary thrombi may occur from stasis }\end{array}$ & No \\
\hline
\end{tabular}

Data from [27]. 


\begin{tabular}{lccc} 
TABLE 2 Improvements in functional parameters following pulmonary endarterectomy & \\
& Pre-operative & 3-month follow-up & p-value \\
\hline Patients $\mathbf{~}$ & 314 & 306 & \\
mPAP $\mathbf{~ m ~ m g ~}$ & $48 \pm 12$ & $26 \pm 10$ & $<0.001$ \\
PVR dyn $\mathbf{s} \cdot \mathbf{c m}^{-\mathbf{5}}$ & $805 \pm 365$ & $301 \pm 232$ & $<0.001$ \\
Cardiac index L· $\mathbf{m i n}^{-\mathbf{1}} \cdot \mathbf{m}^{-\mathbf{2}}$ & $2.0 \pm 0.7$ & $2.5 \pm 0.5$ & $<0.001$ \\
6MWD $\mathbf{m}$ & $269 \pm 119$ & $367 \pm 108$ & $<0.001$ \\
NYHA-FC I/II \% & 8.7 & $83.0^{\#}$ & \\
\hline
\end{tabular}

mPAP: mean pulmonary arterial pressure; PVR: pulmonary vascular resistance; 6MWD: 6-min walking distance; NYHA-FC: New York Heart Association functional class. ${ }^{\#}$ : $87.5 \%$ at 12 months. Reproduced and modified from [29] with permission from the publisher.

\section{Survival after PEA}

Prior to the development of PEA there were no treatments available for CTEPH and consequently survival of patients with CTEPH was poor. Historically, a mean survival of 6.8 years was reported, and in patients with $\mathrm{mPAP}$ of $\geqslant 50 \mathrm{mmHg}$, 3-year mortality was $\sim 90 \%[31,32]$. Survival is best in patients with surgically accessible disease undergoing PEA, but there are no randomised controlled trials and few comparisons with patients managed medically. In a cohort of patients from the UK, there was significantly superior mid-term survival in patients treated with PEA, compared with best medical management at the time (treatment with a prostanoid, endothelin receptor antagonist or phosphodiesterase type 5 inhibitor; $\mathrm{p}=0.023$ ) [33]. In-hospital survival following PEA is related to the level of experience of the surgical team and the PVR at baseline. In the latest retrospective case series from UCSD (the most experienced PEA centre), an in-hospital mortality of $2.2 \%$ in the last 500 consecutive cases was reported, compared with $5.2 \%$ for the preceding 1000 cases, highlighting the importance of experience of the CTEPH team in reducing mortality following PEA [4]. In the prospective European CTEPH registry, which included some centres with a lower patient volume and less experience, overall in-hospital mortality was 4.7\% [18].

Operative mortality increases with increasing pre-operative PVR [5, 15], and may be five- to 10 -fold higher in patients with pre-operative PVR $>1200 \mathrm{dyn} \cdot \mathrm{s} \cdot \mathrm{cm}^{-5}$ [5]. However, good outcomes can be achieved in patients with PVR $>1000 \mathrm{dyn} \cdot \mathrm{s} \cdot \mathrm{cm}^{-5}$, some of whom have the most to gain from surgery despite higher risk, and so there are no upper limits of PVR, mPAP or degree of right ventricular dysfunction that preclude surgery [13]. There are fewer data on longer-term outcomes. A prospective study evaluating patients for up to 5 years following PEA reported cumulative survival of $84 \%$ at 5 years (95\% CI 76-89\%) [30]. The Papworth group has a follow-up programme for all patients after PEA and reported a 5-year survival of $92.5 \%$, conditional from 3-month follow-up, indicating that in patients who survive the peri-operative period, the medium-term outcome is excellent [34]. In a longer-term study of 77 patients, freedom from disease-specific death at 5 and 10 years post-PEA was determined to be $84 \%$ and $82 \%$, respectively, although the cohort number was small at later follow-up [35].

\section{Persistent PH following PEA}

Incidence and causes of persistent PH

Evidence suggests that up to one-third of patients may have persistent (or residual) $\mathrm{PH}$, despite apparently successful PEA surgery $[15,19,36]$. The actual incidence is difficult to quantify as many centres do not routinely re-investigate all patients with right heart catheterisation following PEA surgery and those that do have used varying criteria to define persistent $\mathrm{PH}$. The incidence and severity are dependent upon the pre-operative pressure and distribution of disease. Persistent $\mathrm{PH}$ may be caused by concomitant small-vessel arteriopathy (second compartment) in patients with operable proximal disease, which is challenging to determine prior to surgery [37]. Persistent $\mathrm{PH}$ can also result from failure to surgically remove more distal chronic thromboembolic disease by inexperienced surgeons. Recurrent $\mathrm{PH}$ is less common and of different aetiology and is caused by a further thromboembolic episode after a successful PEA clearance and a confirmed reduction in PH post-PEA. Recurrent PH may be defined according to mPAP or PVR but, as is the case in persistent PH, there is no consensus on the definition.

\section{Management of persistent PH}

Treatment of persistent PH post-PEA is challenging in the operating room and intensive care unit. Some patients may experience difficulty weaning from $\mathrm{CPB}$ and have right heart failure and low cardiac output. Additionally, the risk of reperfusion injury is higher and further compounds the problem. ECMO has been 
used to support and salvage patients with persistent $\mathrm{PH}$ and severe compromise after surgery, as well as patients with severe reperfusion lung injury. Veno-arterial ECMO is necessary if there is haemodynamic instability, particularly in cases of persistent $\mathrm{PH}$, while veno-venous ECMO is sufficient for reperfusion injury alone [2]. ECMO has also been used to provide haemodynamic support in patients with right ventricular failure [38]. The period of support has ranged from 48 to $359 \mathrm{~h}$, with a median duration of 5 days and survival of up to $57 \%$ [38]. It is recommended that all specialist PEA centres have ECMO capability to cope with the most severe complications after surgery [2]. Pharmacological therapy to decrease PVR may be a supportive option in the post-operative management of persistent PH, although evidence is limited to small studies. For example, a study of 22 patients undergoing PEA who were randomised to receive either a single dose of inhaled iloprost or normal saline post-operatively found that iloprost improved cardiac index while reducing mPAP and PVR, in comparison with saline [39].

Medical treatment may be considered an effective option in some patients with persistent $\mathrm{PH}$ who have ongoing functional limitation following PEA. The drug riociguat was approved by the European Commission in March 2014 for use in patients with persistent or recurrent PH after PEA [40].

\section{Survival following persistent $\mathrm{PH}$}

Despite its challenges, some evidence suggests that moderate persistent $\mathrm{PH}$ after PEA in patients who survive surgery may have a limited effect on medium-term survival. In a study of 314 patients who survived PEA surgery and completed 3 months' follow-up, $31 \%$ had persistent PH as defined by mPAP of $\geqslant 30 \mathrm{mmHg}$ [29], with the threshold for definition derived from the cohort of RiEDEL et al. [31], because $30 \mathrm{mmHg}$ determined impaired survival in untreated patients. At 3 months after surgery, 210 patients had mPAP $<30 \mathrm{mmHg}$ and $96 \mathrm{had} \mathrm{mPAP} \geqslant 30 \mathrm{mmHg}$, which had an impact upon New York Heart Association functional class and therefore upon quality of life [29]. However, after 5 years, conditional survival was $90 \%$ and did not differ between groups [29].

There is good evidence to indicate that patients with persistent PH immediately following PEA have an increased risk of in-hospital death. The first study to clearly demonstrate this was from UCSD, and showed that there was a threshold effect for a safe level of post-operative PVR. The mortality rate for patients with a post-operative PVR $>500 \mathrm{dyn} \cdot \mathrm{s} \cdot \mathrm{cm}^{-5}$ was $31 \%$ versus $0.9 \%$ in patients with PVR $<500 \mathrm{dyn} \cdot \mathrm{s} \cdot \mathrm{cm}^{-5}$ [15]. Similarly, the large prospective, multicentre CTEPH registry found that mortality post-PEA was highly related to PVR after surgery [18].

\section{Management of inoperable thromboembolic disease}

The European CTEPH registry indicated that up to $37 \%$ of patients with diagnosed CTEPH were considered inoperable, although this varied with centre experience and also the denominator of the type of patient referred $[6,18]$. As mentioned, reasons for a decision of inoperability included "distal disease" (inaccessible thromboembolism seen on imaging and unseen distal arteriopathy accounting for too high a proportion of the PVR), comorbidity and the presence of certain risk factors. Approximately $48 \%$ of inoperable patients with CTEPH had distal disease [6]. The presence of comorbidities accounted for approximately $13 \%$ of inoperable cases [6]. Patients whose elevated PVR is a result of small-vessel arteriopathy may not experience a significant decrease in PVR after PEA, increasing their peri-operative risk [10]. In approximately $10 \%$ of patients, the reason that they were classed as inoperable was a significantly elevated PVR alone [6]. If patients are not considered candidates for PEA, or refuse surgery, targeted pharmacological treatments may be considered [40].

\section{Conclusions}

Surgery is currently the only definitive therapy with proven curative potential in CTEPH. The beneficial effects of decreased PVR and MPAP can be immediate and dramatic, and translate into improved symptoms and survival [8]. PEA should be considered in all patients with CTEPH, with evaluation and operation at experienced centres, by expert surgeons, using recommended diagnostic algorithms [2]. Survival after PEA is good when performed at expert centres, with low in-hospital mortality and preserved cognitive function despite DHCA. ECMO is useful to help salvage patients with the most severe complications following PEA. However, not all patients with CTEPH are considered operable and some may have persistent $\mathrm{PH}$ following surgery. These patients may be candidates for medical treatment.

\section{Acknowledgements}

Editorial assistance was provided by Adelphi Communications Ltd (Bollington, UK), supported by Bayer Pharma AG.

\section{References}

1 Galiè N, Hoeper MM, Humbert M, et al. Guidelines for the diagnosis and treatment of pulmonary hypertension. Eur Respir J 2009; 34: 1219-1263. 

2013; 62: Suppl. 25, D92-D99.

3 Hoeper MM, Madani MM, Nakanishi N, et al. Chronic thromboembolic pulmonary hypertension. Lancet Respir Med 2014; 2: 573-582.

4 Madani MM, Auger WR, Pretorius V, et al. Pulmonary endarterectomy: recent changes in a single institution's experience of more than 2,700 patients. Ann Thorac Surg 2012; 94: 97-103.

5 Dartevelle P, Fadel E, Mussot S, et al. Chronic thromboembolic pulmonary hypertension. Eur Respir J 2004; 23 : 637-648.

6 Pepke-Zaba J, Delcroix M, Lang I, et al. Chronic thromboembolic pulmonary hypertension (CTEPH): results from an international prospective registry. Circulation 2011; 124: 1973-1981.

7 Hoey ET, Mirsadraee S, Pepke-Zaba J, et al. Dual-energy CT angiography for assessment of regional pulmonary perfusion in patients with chronic thromboembolic pulmonary hypertension: initial experience. AJR Am J Roentgenol 2011; 196: 524-532.

8 Fedullo P, Kerr KM, Kim NH, et al. Chronic thromboembolic pulmonary hypertension. Am J Respir Crit Care Med 2011; 183: 1605-1613.

9 Taboada D, Pepke-Zaba J, Jenkins DP, et al. Outcome of pulmonary endarterectomy in symptomatic chronic thromboembolic disease. Eur Respir J 2014; 44: 1635-1645.

10 Banks DA, Pretorius GV, Kerr KM, et al. Pulmonary endarterectomy: part I. Pathophysiology, clinical manifestations, and diagnostic evaluation of chronic thromboembolic pulmonary hypertension. Semin Cardiothorac Vasc Anesth 2014; 18: 319-330.

11 Jensen KW, Kerr KM, Fedullo PF, et al. Pulmonary hypertensive medical therapy in chronic thromboembolic pulmonary hypertension before pulmonary thromboendarterectomy. Circulation 2009; 120: 1248-1254.

12 Ghofrani HA, D'Armini AM, Grimminger F, et al. Riociguat for the treatment of chronic thromboembolic pulmonary hypertension. N Engl J Med 2013; 369: 319-329.

13 Banks DA, Pretorius GV, Kerr KM, et al. Pulmonary endarterectomy: part II. Operation, anesthetic management, and postoperative care. Semin Cardiothorac Vasc Anesth 2014; 18: 331-340.

14 Shenoy V, Anton JM, Collard CD, et al. Pulmonary thromboendarterectomy for chronic thromboembolic pulmonary hypertension. Anesthesiology 2014; 120: 1255-1261.

15 Jamieson SW, Kapelanski DP, Sakakibara N, et al. Pulmonary endarterectomy: experience and lessons learned in 1,500 cases. Ann Thorac Surg 2003; 76: 1457-1462.

$16 \mathrm{Ng} \mathrm{C}$, Jenkins DP. Surgical management of chronic thromboembolic pulmonary hypertension. Br J Hosp Med 2013; 74: 31-35.

17 Kerr KM, Auger WR, Marsh JJ, et al. Efficacy of methylprednisolone in preventing lung injury following pulmonary thromboendarterectomy. Chest 2012; 141: 27-35.

18 Mayer E, Jenkins D, Lindner J, et al. Surgical management and outcome of patients with chronic thromboembolic pulmonary hypertension: results from an international prospective registry. J Thorac Cardiovasc Surg 2011; 141: 702-710.

19 Thistlethwaite PA, Madani MM, Kemp AD, et al. Venovenous extracorporeal life support after pulmonary endarterectomy: indications, techniques, and outcomes. Ann Thorac Surg 2006; 82: 2139-2145.

20 Gan HL, Zhang JQ, Sun JC, et al. Preoperative transcatheter occlusion of bronchopulmonary collateral artery reduces reperfusion pulmonary edema and improves early hemodynamic function after pulmonary thromboendarterectomy. J Thorac Cardiovasc Surg 2014; 148: 3014-3019.

21 Jenkins DP, Madani M, Mayer E, et al. Surgical treatment of chronic thromboembolic pulmonary hypertension. Eur Respir J 2013; 41: 735-742.

22 Thomson B, Tsui SS, Dunning J, et al. Pulmonary endarterectomy is possible and effective without the use of complete circulatory arrest - the UK experience in over 150 patients. Eur J Cardiothorac Surg 2008; 33: 157-163.

23 Vuylsteke A, Sharples L, Charman G, et al. Circulatory arrest versus cerebral perfusion during pulmonary endarterectomy surgery (PEACOG): a randomised controlled trial. Lancet 2011; 378: 1379-1387.

24 Morsolini M, Nicolardi S, Milanesi E, et al. Evolving surgical techniques for pulmonary endarterectomy according to the changing features of chronic thromboembolic pulmonary hypertension patients during 17-year single-center experience. J Thorac Cardiovasc Surg 2012; 144: 100-107.

25 Baglin TP, Brush J, Streiff M. Guidelines on use of vena cava filters. Br J Haematol 2006; 134: 590-595.

26 Jamieson SW, Kapelanski DP. Pulmonary endarterectomy. Curr Probl Surg 2000; 37: 165-252.

27 Lang IM, Madani M. Update on chronic thromboembolic pulmonary hypertension. Circulation 2014; 130: 508-518.

28 D’Armini AM, Morsolini M, Mattiucci G, et al. Pulmonary endarterectomy for distal chronic thromboembolic pulmonary hypertension. J Thorac Cardiovasc Surg 2014; 148: 1005-1011.

29 Freed DH, Thomson BM, Berman M, et al. Survival after pulmonary thromboendarterectomy: effect of residual pulmonary hypertension. J Thorac Cardiovasc Surg 2011; 141: 383-387.

30 Corsico AG, D’Armini AM, Cerveri I, et al. Long-term outcome after pulmonary endarterectomy. Am J Respir Crit Care Med 2008; 178: 419-424.

31 Riedel M, Stanek V, Widimsky J, et al. Longterm follow-up of patients with pulmonary thromboembolism. Late prognosis and evolution of hemodynamic and respiratory data. Chest 1982; 81: 151-158.

32 Kunieda T, Nakanishi N, Satoh T, et al. Prognoses of primary pulmonary hypertension and chronic major vessel thromboembolic pulmonary hypertension determined from cumulative survival curves. Intern Med 1999; 38: 543-546.

33 Condliffe R, Kiely DG, Gibbs JS, et al. Improved outcomes in medically and surgically treated chronic thromboembolic pulmonary hypertension. Am J Respir Crit Care Med 2008; 177: 1122-1127.

34 Freed DH, Thomson BM, Tsui SS, et al. Functional and haemodynamic outcome 1 year after pulmonary thromboendarterectomy. Eur J Cardiothorac Surg 2008; 34: 525-529.

35 Ishida $\mathrm{K}$, Masuda $\mathrm{M}$, Tanabe $\mathrm{N}$, et al. Long-term outcome after pulmonary endarterectomy for chronic thromboembolic pulmonary hypertension. J Thorac Cardiovasc Surg 2012; 144: 321-326.

36 Bonderman D, Skoro-Sajer N, Jakowitsch J, et al. Predictors of outcome in chronic thromboembolic pulmonary hypertension. Circulation 2007; 115: 2153-2158. 
37 Kim NH, Fesler P, Channick RN, et al. Preoperative partitioning of pulmonary vascular resistance correlates with early outcome after thromboendarterectomy for chronic thromboembolic pulmonary hypertension. Circulation 2004; 109: 18-22.

38 Berman M, Tsui S, Vuylsteke A, et al. Successful extracorporeal membrane oxygenation support after pulmonary thromboendarterectomy. Ann Thorac Surg 2008; 86: 1261-1267.

39 Kramm T, Eberle B, Guth S, et al. Inhaled iloprost to control residual pulmonary hypertension following pulmonary endarterectomy. Eur J Cardiothorac Surg 2005; 28: 882-888.

40 Hoeper MM. Pharmacological therapy for patients with chronic thromboembolic pulmonary hypertension. Eur Respir Rev 2015; 24: 272-282. 